\title{
EXERCÍCIO FÍSICO E PLASTICIDADE NEUROGÊNICA: BENEFÍCIOS RELACIONADOS ÀS FUNÇÕES MENTAIS DO IDOSO
}

\author{
Vernon Furtado da Silva \\ Coordenador do Laboratório de Neuromotricidade e Performance Motora (LANPEM)/UERJ/RJ \\ vernonfurtado2005@yahoo.com.br
}

\section{Luis Henrique Brandão Ribeiro}

Doutorando na Universidade Trás Montes e Alto Douro/Portugal

Luiz.henrique@highlight.tur.br

\section{Rodolfo de Alkmim Moreira Nunes}

Pesquisador do Laboratorio de Neuromotricidade e performance Motora(LANPEM)/UERJ/RJ

\section{Juliana Cavalcante}

Mestranda em Neurociência pela Universidade de La República/UdelaR

j.cavalcante-@hotmail.com

\section{Maria Auxiliadora Freire Siza \\ Doutoranda em Neurociência pela Universidade de La República/UdelaR \\ asiza@bol.com.br}

\author{
Mauricio Rocha Calomeni \\ Doutorando no IPUB/UFRJ/RJ \\ mauriciocalomeni@gmail.com
}

\section{RESUMO}

Relaciona alguns comprovados efeitos do exercício sobre vários fatores do bem estar físico e mental do idoso, buscando-se evidenciar estes benefícios com mudanças cerebrais vistas a partir de uma série de processos e mecanismos específicos. Estabelece-se uma inter-relação destes efeitos com funções bio-estruturais e biooperacionais do cérebro sobre as ineficiências comportamentais advindas do processo de envelhecimento e problemas orgânicos a ele relacionados. Busca-se mostrar todo e qualquer comportamento como resultado de processos neurotransmissores integrando-se em ordem funcional. Analisa-se a neurogêse como o principal fenômeno da plasticidade cerebral, visualizando-a como uma referência para a formulação de métodos e técnicas de reabilitação de várias doenças e desordens do comportamento motor e cognitivo, principalmente algumas relacionadas ao processo de envelhecimento. Evidências que dão suporte a discussão e andamento do trabalho advêm de pesquisas realizadas com humanos e não humanos. Estas últimas estão mostradas em um maior percentual.

Palavras-chave: Funções bio-estruturais, funções bio-operacionais, plasticidade cerebral, processo de envelhecimento. 


\section{INTRODUÇÃO}

A verificação do impacto, da atividade física regular, sobre fatores de saúde, prevenção de doenças e melhora de estados doentios tais como doenças coronarianas, estados patogênicos oriundos de diabetes e obesidade têm, ao longo de alguns anos, permeado a maioria da pesquisa nesta linha. Devido ao alto custo e o alto índice de morbidade social, produzidos por casos de desordens mentais, de certa forma vêm direcionando pesquisas para um enfoque mais relacionado ao contexto entre a atividade física e o tratamento destas desordens e alternativamente buscando verificar os seus efeitos ou eficácia, sobre auto-estima, depressão, ansiedade, doenças do sono, resistência ao estresse e outras que sobremaneira afetam uma grande parte da população idosa (infligindo também adultos e, inclusive, jovens indivíduos). De certa forma, estes estudos têm adentrado a verificação de parte destes estados mórbidos, em uma variedade de fatores de cognição e demência associadamente ao mal de Parkinson, uma doença que afeta diretamente as funções cognitivas do seu portador. Apesar do robusto número de evidências que atribui, ao exercício físico, a condição de interferir positivamente sobre vários estados móbidos da saúde cerebral, alguns estudos contrariam esta possível e relevante possibilidade.

Todavia, um número considerável de pesquisas visualizadas sobre as mudanças que os exercícios regulares produzem diretamente sobre o cérebro acrescenta bases de certeza quanto a tal benefício. Estas pesquisas estão relacionadas tanto com mudanças em tecido neural e mecanismos de neurotransmissão, quanto à neurogênese, uma forma plástica do cérebro que possibilita a produção de novos neurônios. Esta última, podendo ser pensada como um dos mais relevantes processos neuroplástico que o sistema nervoso possa mediar em momentos retrogênicos da vida humana.

\section{DESENVOLVIMENTO}

\subsection{Exercício e cognição em idosos}

Um grande número de pesquisa tem promovido suporte para os benefícios de exercícios regulares, tanto em termos de cognição, especificamente falando, quanto de fatores que minimizam funções cognitivas em idosos.

Em um estudo sob um modelo de meta-análise, englobando uma grande variedade de pesquisa, Netz e associados (2005) evidenciaram estes benefícios sobre um grande montante de fatores de bem estar mental e físico, de certa forma associados a tarefas de níveis de cognição. Mais especificamente, Colcombe e Kraemer (2003), também em um trabalho de verificação meta analítica mostraram que o treinamento aeróbico de intensidade moderada está estritamente ligado a melhoras em certos fatores de cognição, principalmente nos relativos ao controle executivo da mente. Ou seja, tarefas que demandam alta análise em construção de eventos sequenciais. Estes mesmos autores também mostraram que o referido treinamento pode ter tido efeitos anxiolítico nos grupos estudados, moderando níveis de depressão e mediando níveis altos de estresse no grupo estudado. A eficácia de um treinamento aeróbio, desenvolvido entre 2 e 4 meses, evidenciou seus benefícios como aproximados aos conseguidos em função da estabilização mediada por efeitos de anti-depressantes, sendo que quando o exercício praticado se caracteriza por um alto nível de compleição, os resultados se tornam mais efetivos (SINGH e ASSOCIADOS, 2005).

Resultados parecidos aos de Netz e associados (2005) foram publicados por outros autores, como por exemplo, Salmon (2001) em uma coleção de estudos longitudinais (e alguns transversais), mostrando também as benesses do treinamento aeróbico como mediador efetivo de depressão e proteção contra as conseqüências danosas que o estresse pode produzir em idosos sedentários. Antunes e colaboradores (2001) reportaram significativas melhoras em fatores de cognição, incluindo memória e atenção, em mulheres com idade entre 60 e 70 anos, mas não em idosas sedentárias na mesma média de idade. As atividades intervenientes incluíram 60 minutos de caminhada e exercícios de alongamento e flexibilidade.

Persp. online: biol. \& saúde, Campos dos Goytacazes, 6 (2), 35-43, 2012 
Em estudos baseando-se em nível de atividade física anterior, tem se observado uma relação inversa entre tempo de atividade e declínio cognitivo. Yaffe e colegas (2001), verificaram que o nível de performance cognitiva de quase 6.000 mulheres com 65 anos ou mais era proporcionalmente melhor em relação ao maior montante de atividade desenvolvida, em um período entre 6 e 8 anos de observação. Também, Dik e associados (2003) verificaram em um número de indivíduos estudados e com idade entre 62 e 85 anos, que níveis moderados e consistentes de atividade física beneficiava a performance destes em tarefas de processamento mental requerendo rapidez de resposta. O período associado de atividade física se enquadrava entre os 15 e 25 anos destes, verificando-se ainda que as mulheres participantes do mesmo estudo não demonstraram relação considerável entre atividade física anterior e o mesmo tipo de teste, nem tampouco, outros homens cujas atividades no mesmo período eram desempenhadas em nível alto de exigência.

Analisado em estudos vinculados aos seus efeitos em relação a doenças neurodegenerativas, o exercício físico tende a ser explicado como de certa forma exercendo efeito de proteção e, ainda, revelando poder de estabilização em declínio cognitivo, mesmo quando o processo de deterioração se tenha iniciado (PODEWILS e ASSOCIADOS 2005 e LARSON e ASSOCIADOS, 2006). Estes autores confirmam que estes tipos de benefícios podem se extender, além da doença de Alzheimer (DA), também a outros tipos de doenças tais como a de Huntington e a de Parkinson. Em portadores de Alzheimer, outras pesquisas indicam que minimização em depressão, diminuição em declínio cognitivo e aumento em competência física podem ser conseguidos quando os mesmos adentram um programa de exercícios adequados (TERI e ASSOCIADOS 2003). Em outro estudo tido como de valiosa contribuição para o entendimento sobre a função do exercício em casos de neurodegeneração, Róvio e colegas (2005) associaram variáveis tais como tempo de lazer e exercício físico, verificando que esta atividade pode fazer prevenir contra o processo de demência, mesmo quando a esta se inicia em um determinado período da meia idade. Inclusive, em portadores do gene apoeE4, cujos portadores são indivíduos altamente suscetíveis ao desenvolvimento da DA.

Todos os resultados mostrados acima possuem referências para o desenvolvimento e aceitação da noção de que exercício e atividade física podem impulsionar funções cognitivas em idosos, efeitos anxiolíticos, minimização em índices de depressão, bem como pode promover uma gradual resistência contra os efeitos das doenças degenerativas sobre o cérebro.

Em assim sendo, a quais tipos de mudanças no cérebro, estariam tais mudanças funcionais relacionadas? A resposta a tal indagação pode ser parcialmente respondida em alusão a duas principais mudanças. Uma associada a funções bio-estruturais e a outra, a funções bio-operacionais do cérebro. Esta última ligando-se a velocidade de processamento mental, estimulada por melhoras da circulação sanguínea no cérebro e, além disso, alteração na síntese de neurotransmissores específicos bem como em ajustes na degradação destes. Provavelmente, fatores adicionais inerentes à função sanguínea tais como a diminuição da pressão arterial, concomitâncias de equilíbrio em triglicerídios no plasma sanguíneo e dispersão dos agentes neuroquímicos malévolos que facilitam a formação de placas bloqueadoras do seu fluxo são algumas das principais alterações que podem beneficiar a capacidade funcional do idoso (e mesmo de indivíduos jovens). Tudo isso podendo se refletir na qualidade de vida e longevidade dos mesmos (VAN BOXTEL ET AL, 1997 e WOOD ET AL, 1999).

Pode se ver assim que existe uma grande quantidade de estudos a qual claramente demonstram ser a prática adequada de exercício (físico e/ou mental) um fator de melhora e fortalecimento de uma série de funções cognitivas, dentre as quais se incluem atenção, memória, percepção e habilidades de processamento mental. E embora as evidências possam vir, em sua maioria, de exercícios de natureza aeróbia, outros de ordem anaeróbias podem também ser benéficos. Percebe-se, também, que o maior impacto destes exercícios refere-se à capacidade de controle executivo da mente, uma condição de referência bio-operacional, considerando-se a efetividade participativa da mente sobre a execução dos mesmos. Muito embora não se tenha dialogado aqui sobre possíveis controvérsias, um considerável número de estudos tem falhado em revelar reais benefícios da atividade física sobre funções cognitivas de idosos.

\subsection{Mudanças bio-estruturais permeadas pelo exercício físico no cérebro do idoso.}

Persp. online: biol. \& saúde, Campos dos Goytacazes, 6 (2), 35-43, 2012 37

seer.perspectivasonline.com.br 
Como afirmam alguns neurocientistas, muito mais do que já se pensava antes, os benefícios do exercício, inclusive através de prática mental, vão muito além dos resultados vistos em relação a fatores periféricos à saúde geral.

$\mathrm{Na}$ verdade, "pensar o exercício" poderia ser visto como um direcionamento cultural quando analisado em relação a certas culturas orientais, mas não tanto em referência às ocidentais. Não obstante uma gama de estudos, em adaptações neurais, ter mostrado que um alto nível de esforço mental sobre um exercício tende a ser bem mais efetivo quando comparado ao mesmo exercício, mas praticado sem uma devida participação focal. Esta prática, todavia, não parece ser ainda percebida em algumas culturas. De fato, um grande "bulk" de evidências mostra, por exemplo, que o simples ato de pensar o movimento de um braço (em uma série de repetições) é suficiente para promover ganhos significativos em força. Considerando-se a importância deste fator orgânico na vida cotidiana ou especial do ser humano, esta possibilidade pode ser vista como uma grande benesse, principalmente para indivíduos idosos cuja manutenção de um estado adequado de força diminui radicalmente com o avançar da idade. Por exemplo, em um estudo cujo exercício era voltado à hipertrofia do músculo trapésio, Yue e colegas (1992) mostraram em 36 voluntários, entre 65 e 93 anos de idade, todos saudáveis, que um pequeno esforço mental, comparado a um grande esforço nesta linha (cerca de $30 \%$ de um possível $100 \%$ de uma contração), promoveram ganhos bem diferenciados. Ou seja, sendo em termos de apenas $3 \%$ no grupo que enquanto praticava o exercício permanecia assistindo um programa de televisão (baixo esforço mental) e, de $15 \%$, no que ao praticar o exercício, empreendia um alto montante de esforço mental sobre o mesmo. Esta proporcionalidade foi mostrada através de EEG de potencial relacionado, indicando que um esforço mental significativo, aumenta a produção de sinais neurais para o músculo, enquanto que um esforço mental menor, não o faz.

Ainda nesta linha de investigação, outros pesquisadores têm mostrando que apenas o pensar um braço em contração, sem mesmo a produzir, pode promover ganhos consideráveis em melhoria de força.

Mesmo ao se considerar que o treinamento mental deva ser organizado e praticado em função da sua grande valia e principalmente pensando-se sobre doenças neuromusculares ou impedimento de movimento em condições pós-operatórias, a prática, de exercícios com foco mental restrito tem também se mostrado eficaz em termos de mudanças específicas no cérebro de idosos.

Colcombe e associados (2003), pesquisadores do Instituto Beckman da Universidade de Illinois, verificaram um significativo aumento em volume do cérebro em idosos sedentários que praticaram um programa de atividades aeróbicas, comparados a idosos praticantes de exercícios de flexibilidade. $\mathrm{O}$ aumento foi medido através de imagem por ressonância magnética (MRI) após seis meses do referido programa. Este aumento referiu-se tanto às estimativas da substância branca quanto da cinzenta. Concomitante estimativa, realizada em um grupo controle que foi composto por indivíduos jovens, revelou uma diferença extremamente significativa, visto que neste grupo (não praticante do treinamento) não ocorreu nenhum tipo de ganho. Considerando-se que na literatura correspondente a diminuição do cérebro em idades avançadas é um resultado comumente observado e, quase sempre, correlacionado com baixa funcionalidade cognitiva e saúde cerebral, a acima mencionada eficácia do treinamento aeróbico na minimização do encurtamento do cérebro pode ser efetivamente pensada como também relacionada à prevenção destes fatores deletérios e comuns em períodos de envelhecimento.

O treinamento de base aeróbia tem também se mostrado eficiente em termos da redução e equilíbrio da perda de tecido neural que geralmente também interage com declínios em cognição e memória (Colcombe e associados, 2003), dando substatividade à noção de que o mencionado treinamento vai bem além dos ganhos comuns em termos de saúde cardiovascular. A saúde do cérebro funde-se como um alvo também privilegiado pelas benesses da atividade física, refletindo-se no final, sobre o organismo como um todo.

Outro efeito bio-estrutural do exercício físico sobre o cérebro foi mostrado por Greenough e colegas (1996) ao evidenciarem que o mesmo pode estar associado, em parte, a um aumento em capilares do córtex motor, como visto em estudos realizados com macacos em idade avançada. Considerando-se que este tipo de animal, tem características biológicas bastante aproximadas às de humano, tais resultados servem como uma

Persp. online: biol. \& saúde, Campos dos Goytacazes, 6 (2), 35-43, 2012 
forte evidência científica para o entendimento de que um trabalho aeróbico sistematizado não apenas beneficia os tecidos periféricos do corpo (coração e artérias, por exemplo) do ser humano, mas também repercute diretamente e positivamente na vascularização cerebral do seu praticante. Inclusive, como explicado por aqueles autores, podendo reestruturar tecidos vasculares de várias regiões do cérebro. $\mathrm{O}$ crescimento de bases estruturais para desenvolvimento funcional das regiões cerebrais via exercício físico, foi mostrado também por autores como Van Praag e associados (2005), que evidenciaram o crescimento de uma ampla rede de vasos sanguíneos no hipocampo, sendo que Ding e associados (2006) também mostraram este mesmo crescimento em regiões do córtex. Convém lembrar aqui que bem anteriormente Black e colegas (1990) já tinham identificado um crescimento significativo de conjuntos de vasos sanguíneos no cerebelo de rodentes. Embora estes achados associados ao crescimento de vasos sanguíneos venham de estudos com animais, Pereira e colegas em um estudo recente (2007) verificaram, via a utilização de técnicas por imagem computadorizadas, que um programa com 12 semanas de treinamento cardiovascular foi suficiente para produzir aumento significativo de fluxo sanguíneo no giro-dentado (DG) de indivíduos variando entre 21 e 45 anos de idade. Estes ganhos, como verificados, se relacionaram a melhor performance em tarefas dependentes de habilidades espacial e temporal para suas realizações. Visto que, este aumento deve advir de uma ampliação de vasos e capilares naquele setor neural, pode-se ter este resultado como similar aos anteriores, vistos em espécies diferentes de ratos. Ainda adicionando suporte ao conceito que pesquisas com amostra de não humanos, podem servir de referência para concepção de noções científicas sobre a relação exercício e mudanças neurais em humanos, Colcombe e seus associados (2003), verificaram via técnica de ressonância magnética (MRI), uma significativa diferença entre estruturas cerebrais de idosos com história de longo termo de exercícios de demandas cardiovasculares, comparativamente a idosos tradicionalmente sedentários. Estas diferenças estavam relacionadas principalmente nas regiões frontal e temporal, sites cerebrais que correspondem a funções executivas da mente e memória declarativa, respectivamente.

Um estudo adicional, ainda realizado com ratos do tipo Murine (EHNINGHER e KEMPERMANN, 2003) adicionou dados para os efeitos bio-estruturais do exercício sobre o cérebro, revelados por um aumento substancial de microglias e astrócitos em algumas regiões do neocórtex. E apesar de que as reais funções das microglias e astrócitos nas contingências de produção cerebral tenham, ainda, que ser definidas, para esta data, sabe-se bem que as primeiras promovem um suplemento nutriente das células nervosas do cérebro, bem como participação em composição da energia neural. Estas duas condições podendo ser entendida como de grande importância para o equilíbrio e manutenção da saúde e funcionalidade cerebral.

Mesmo diante da tremenda importância que a literatura atribui aos vários resultados de pesquisas que dão substância à concepção de que um estado "FIT", do organismo como um todo, tende a ter correspondência estreita com bons níveis de atividade física, tal relação não parece, ainda, ser aceita sem restrições. Todavia, a forte concomitância estabelecida entre tais exercícios e neurogenesis, além de promover fortes evidências para a mesma, parece não ter sido questionada por qualquer linha de pesquisa e talvez represente o mais celebrado advento da plasticidade cerebral humana, a qual pode ser induzida por atividade física adequada.

\subsection{Efeito do exercício na neurogêse da retrorgênese e possíveis mecanismos relacionados neurotransmissores.}

Como foi acima apresentada, talvez possa ser da potencial plasticidade neurogênica a qual vários tipos de exercícios físicos têm mostrado influências benéficas, que se defina a chave para a criação de métodos terapêuticos que poderão servir à prevenção e reabilitação de vários transtornos, doenças e desordens neuromusculares hoje tidas como de difícil tratamento. Não tão somente isso, mas podendo-se, ainda, imaginar, que avanços em técnicas aplicadas em buscas de desenvolvimento neurogênico possam vir a servir de base para o tratamento de disfunções neurais tão severas quanto as que se relacionam, por exemplo, ao autismo, considerando-se que este mal está relacionado à incompatibilidade funcional de alguns neurônios frontotemporais (pensados por muitos como sendo inexistentes ou não funcionais em portadores deste mal).

Persp. online: biol. \& saúde, Campos dos Goytacazes, 6 (2), 35-43, 2012 
Fred Gage do Salk Insitute for Biological Studies tem como certa a noção de que o exercício físico ajuda na geração de novas células cerebrais, mesmo em organismo neural de pessoas idosas. A forma indicativa de como novos neurônios podem se formar em decorrência de processos neurogênicos é um fenômeno ainda irrevelado. Principalmente em termos dos mecanismos que possam explicar a sua ordem funcional. Apesar disto, alguns estudiosos neste assunto têm apontado para uma possibilidade de que um maior influxo de cálcio, promovido por níveis do estresse, derivado do exercício em si, possa dar origem à transcrição de certos fatores neurotróficos em determinados neurônios hipocampais. Conforme verificada por Mattson e colegas (2004), esta transcrição define a expressão do fator de gênese conhecido como BDNF (Fator neurotrófico derivado do cérebro) substanciando proteínas que, por sua vez, atuam, na criação neurogênica. Não só em termos desta configuração, o BDNF atua também na proteção de outros neurônios, bem como na estimulação de plasticidade entre sinapses, estas quando associadas a eventos de aprendizagem, atenção e memória, dentre outros.

A plasticidade acima mencionada, não se refere tão somente à proteção e/ou eventos de aprendizagem e memória, mas ainda efeitos em reestruturação celular, como revelada em estudos tais quais os realizados por pesquisadores do medical Research News Center de Boston (2004), comparando certos tipos de ratos ativos a outros sedentários. Depois de produzidas deteriorações em axônios da região ciática destes ratos, os que foram mantidos ativos (em exercício) tiveram restaurados os axônios daquela área em uma significativa maior proporção do que os axônios da mesma área em ratos sedentários. Estes pesquisadores ainda mostraram que a substância neurotrofina foi a grande mediadora da restauração, caracterizando-se como um mecanismo de efetiva reparação dos axônios lesados. Estes resultados não somente devem ser vistos como possivelmente aplicáveis à prevenção de doenças tipos Alzheimer e Parkinson, as quais se caracterizam por perdas gradativas de neurônios, mas também podem se associar à prevenção de atrofia do hipocampo, uma condição comum em ratos idosos (SMALL E ASSOCIADOS, 2002), bem como possibilitar a prevenção da redução em produção neurogênica, uma condição que pode ocorrer tanto em não humanos quanto em humanos idosos sedentários, comparativamente a idosos ativos (KUHN E COLEGAS, 1996 E HEINE E OUTROS, 2004).

Além do aumento em BDNF, observado em topografia hipocampal de cérebros idosos, a plasticidade de outros tipos de neurotransmissores pode também ser influenciada por efeito de exercício. Uma significativa minimização na depleção de dopamina foi constatada por Poulton e Muir (2005) ao examinarem os efeitos do trabalho em esteira rolante de ratos portadores de um determinado estado Parkinsoniano. Também, tem sido visto que o exercício pode induzir aumento em taxa de acetilcolina (FORDYCE E FARRAR, 1991) e serotonina (CARRO ET AL, 2001) em ratos idosos que se exercitam regularmente. Ainda conforme evidenciado por Knusel e associados (1992), o BDNF regula a taxa de certos neurotransmissores no cérebro, como, por exemplo, os que compõem os sistemas colinergético e o dopaminergético, sendo esta regulação um potente mecanismo que se associa às funções de base dos núcleos do complexo pontomesencefalotegmental e da área tegmental com a substância negra. Ou seja, os pontos de projeções difusas dos sistemas colinergético e dopaminergético, respectivamente. Estes mecanismos mediados pela ação do BDNF, induzidos em função de exercícios regulares, configuram as bases para a reestruturação de lesões cerebrais, conforme explicação de Gomes-Pinilla da Universidade da Califórnia (EE. UU).

Um recente estudo realizado por Ploughman e colegas (2009) revelou que exercícios tão simples como o alcançar de um alvo em repetidas tentativas produziu aumento de BDNF e consequentes efeitos deste em ratos que sofreram isquemia focalizada. Em estudo anterior realizado nesta mesma linha, Gómez-Pinilla ET AL (2002) evidenciou os efeitos do exercício em mecanismos inerentes a plasticidade cerebral, via modulation de neurotrophina, em ratos.

Como define Gomez-Pinilla, provavelmente o maior obstáculo na recuperação de lesões no cérebro, seja a dificuldade imposta por alguns substratos químicos desincorporadores funcionais. Estes autores mostram que o exercício pode reduzir esta resistência e fortalecer a produção de fatores neurotróficos que atuam em paralelo sobre outras enzimas eficazes para o crescimento de novos neurônios, bem como para e desenvolvimento funcional de processos neurais que interagem com aprendizagem e memória. Compondo-se, por exemplo, os momentos seguintes a um trauma, o papel destas enzimas pode ser bem ilustrado, visto que o cérebro em seu projeto de prevenção faz aumentar o fluxo de duas proteínas conhecidas pelas abreviaturas de MAG e Nogo-A,

Persp. online: biol. \& saúde, Campos dos Goytacazes, 6 (2), 35-43, 2012 
proteínas estas que têm, paralelamente, o poder químico de inibir o crescimento de novos axônios em neurônios que possam ser deteriorados por um acidente traumático. Via um modelo experimental de deterioração de neurônios hipocampais em ratos, Gómez-Pinilla e associados (2004) expuseram alguns deles para exercícios em uma roda circulante enquanto outros foram mantidos em condição sedentária. Em um espaço de dez dias os níveis de MAG e Nogo-A subiram em termos de $74 \%$ e $59^{\%}$, para as proteínas MAG (Glicoproteína de associação à mielina) e Nogo-A, (Proteína que inibe o crescimento de axônios recém-formados), respectivamente. Este aumento foi verificado no hipocampo dos ratos submetidos à deterioração. Todavia, o efeito quase agudo do exercício na roda circulante, fez diminuir de forma significativa estes índices, mostrandose em termos de $29 \%$ e $17 \%$ a menos nos ratos treinados em comparação aos que não treinaram. Um ponto de destaque para o quadro neuroquímico resultante de um processo de deterioração de nervos cerebrais é o efeito desta deterioração na redução de uma proteína que estimula os efeitos de proteção das células cerebrais por função do BDNF, condição que logo é mediada pelos efeitos direto do exercício em aumento deste fator neurotrófico (BDNF) via regulação do nível de proteína kinase-A (PKA) e desta na redução do efeito da proteína Nogo-A que inibe o crescimento de novos axônios de neurônios danificados experimentalmente. Vê-se aqui, portanto, a natureza de um correlato e amplo mecanismo neuroquímico que se estabelece sob as formas de inibição e estimulação recíproca. Em uma dimensão, reduz o número de proteínas que inibem o crescimento de axônios neuronais em situação pós-trauma e, por outro lado, estimula o aumento de outras proteínas que podem fomentar tal crescimento. De fato, um potencial mecanismo que pode ser explorado em métodos e técnicas de reabilitação voltada ao tratamento de uma série de doenças e estados mórbidos de saúde. Tanto no que se refere ao domínio cognitivo, quanto ao motor e psíquico, os quais interligadamente dão sustentação à vida humana em suas multi-facetas de ligação com o universo.

\section{CONCLUSÕES}

Neste presente trabalho buscou-se evidenciar alguns dos benefícios da atividade física regular sobre vários fatores da saúde humana e sobre o próprio cérebro de indivíduos que a pratica de forma regular. Viu-se que a atividade física, ocorrendo sob uma forma de exercícios aeróbios de intensidade moderada é suficiente para promover alterações em condições mórbidas da mente e do físico. Além de resultar em alterações positivas de desempenho físico e manutenção de saúde, pode incidir na formação de novas redes neuronais em sistemas neurais deteriorados por traumas e outros eventos destrutivos das funções mentais. Os benefícios foram descritos como relacionados a condições básicas de funcionalidade orgânica que incluem o bem estar físico, o mental, controle da depressão, além de proteção contra doenças degenerativas, tais como, as de Alzheimer, Parkinson e demência senil.

\section{REFERÊNCIAS}

ANTUNES HKM, SANTOS RF, HEREDIA RAG, BUENO OFA, MELLO MT. (2001). Alterações cognitivas em idosas decorrentes do exercício físico sistematizado. Revista da Sobama; 6:27-33.

BLACK J.E., ISAACS K.R., ANDERSON B.J., ALCANTARA A.A., GREENOUGH W.T. (1990) Learning causes synaptogenesis, whereas motor activity causes angiogenesis, in cerebellar cortex of adult rats. Proc. Natl. Acad. Sci. 87:5568-5572

CARRO, E., TREJO, L. J., BUSIGUINA, S. \& TORRES, ALEMAN, I. (2001). Circulating insulin-like growth factor 1 mediates the protective effects of physical exercise against brain insults of different etiology and anatomy. J. Neurosci., 21, 5678-5684.

COVALT ANN, M.A. (2006). Benefits of Exercise include Protection from Brain Diseases and Aging, New research Online. http://hdlighthouse.org/treatment- care/care/hdltriad/exercise/updates/1312exercise.php.

Persp. online: biol. \& saúde, Campos dos Goytacazes, 6 (2), 35-43, 2012 
DIK, M.G., DEEG, D.J.H., VISSER, M. \& JONKER, C. (2003). Early life activity and cognition in old age. J. Clin. Exp. Neuropsychol., 25, 643-653.

ERIC B. LARSON, E.B; WANG, L.; BOWEN, J.D.; MCCORMIC, TERI, L.; CRANE, P. AND KUKULL, W. (2006). Exercise Is Associated with Reduced Risk for Incident Dementia among Persons 65 Years of Age and Older. Annals of International medicine. 144, 2, 73-81.

FORDYCE, D.E. \& FARRAR, R.P. (1991). Enhancement of spatial learning in F344 rats by physical activity and related learning-associated alterations in hippocampal and cortical cholinergic functioning. Behav Brain Res., 46, 123-133

GÓMEZ-PINILLA, FERNANDO, ZHE YING, ROLAND R. ROY, RAFFAELLA MOLTENI, AND V. REGGIE EDGERTON (2002). Voluntary Exercise Induces a BDNF-Mediated Mechanism That Promotes Neuroplasticity. J. Neurophysiol. 88: 2187-2195.

HEINE VM, MASLAM S, JOELS M, LUCASSEN PJ. (2004). Prominent decline of newborn cell proliferation, differentiation, and apoptosis in the aging dentate gyrus, in absence of an age-related hypothalamuspituitaryadrenal axis activation. Neurobiol Aging 25:361-375.

KNUSEL, B., WINSLOW, J.W., ROSENTHAL., A., BURTON, L.E., SEID, D.P., NIKOLICS, K., HEFTI, F. (1992). Promotion of central cholinergic and dopaminergic neuron differentiation by brain-derived neurotrophic factor but not neurotrophin 3. Proc Natl

KUHN HG, DICKINSON-ANSON H, GAGE FH (1996) Neurogenesis in the dentate gyrus of the adult rat: age-related decrease of neuronal progenitor proliferation. J Neurosci 16:2027-2033.

LINDA TERI, PHD; LAURA E. GIBBONS, PHD; SUSAN M. MCCURRY, PHD; REBECCA G. LOGSDON, PHD; DAVID M. BUCHNER, MD; WILLIAM E. BARLOW, PHD; WALTER A. KUKULL, PHD; ANDREA Z. LACROIX, PHD; WAYNE MCCORMICK, MD, MPH; ERIC B. LARSON, MD, MPH. (2003). Exercise Plus Behavioral Management in Patients With Alzheimer Disease. JAMA. 290 (15):2015-2022.

MARK P. MATTSON, WENZHEN DUAN, RUQIAN WAN, AND ZHIHONG GUO. (2004). Prophylactic Activation of Neuroprotective Stress Response Pathways by Dietary and Behavioral Manipulations. NeuroRx, January, 1 (1), 111-116.

MEDICAL RESEARCH NEWS (Online). (2004). Exercise can help brain healing process. 2, June, 21:14.

MEDICAL RESEARCH NEWS (Online). (2004). Exercise protects brain cells affected by Parkinson's, 24. October, 23:18

MICHELLE PLOUGHMAN, PHD; VICTORIA WINDLE, PHD; CRYSTAL L. MACLELLAN, PHD; NICOLE WHITE, BSC; JULES J. DORÉ, PHD DALE CORBETT, PHD (2009). Brain-Derived Neurotrophic Factor Contributes to Recovery of Skilled Reaching After Focal Ischemia in Rats. Stroke, 40, 1490.

NETZ Y, WU MJ, BECKER BJ, TENENBAUM G. (2005). Physical activity and psychological well-being in advanced age: a meta-analysis of intervention studies. Psychol Aging, 20: 272-284.

PEREIRA, A.C.; HUDDLESTON, D.E.; BRICKMAN, A.M.; SOSUNOV, A.A.; HEN, R.; MCKHANN, G.M.; SLOAN, R.; GAGE, F.H.; BROWN, T.R. AND SMALL, S.A. (2007). An in vivo correlate of exerciseinduced neurogenesis in the adult dentate gyrus. Proc. Natl. Acad. Sci. (USA), 104, 5638-5643.

Persp. online: biol. \& saúde, Campos dos Goytacazes, 6 (2), 35-43, 2012 
PLOUGHMAN, M; VICTORIA WINDLE, V.; MACLELLAN, C. L. ; WHITE, N. ; JULES J. DORÉ, J.J.; CORBETT, D. Brain-Derived Neurotrophic Factor Contributes to Recovery of Skilled Reaching After Focal Ischemia in Rats.

PODEWILS, L. J., GUALLAR, E., KULLER, L. H., FRIED, L. P., LOPEZ, O., CARLSON, M., et al. (2005). Physical activity, APOE genotype, and dementia risk: Findings from the Cardiovascular Health Cognition Study. American Journal of Epidemiology, 161,639-651

POUTLTON, N.P.; MUIR, G.D. (2005). Treadmill training ameliorates dopamine loss but not behavioral deficits in hemi-parkinsonian rats. Exp Neurol., 193, 181-197.

ROVIO S, KÅREHOLT I, HELKALA EL, VIITANEN M, WINBLAD B, TUOMILEHTO J, SOININEN H, NISSINEN A, KIVIPELTO M.(2005). Leisure-time physical activity at midlife and the risk of dementia and Alzheimer's disease. Lancet Neurol. Nov ;4(11):690-1.

SALMON, P. (2001). Effects of physical exercise on anxiety, depression and sensitivity to stress: a unifying theory. Clin Psychol Rev. 21: 33-61.

SINGH, N.A. e associados (2005). A randomized contolled trial of high versus low intensity weight strenght training versus general practicioner care for clinical depression in older adults. J. Gerontol. A Biolo. Sic. Med. Sci. 60, 768-776.

SMALL SA, TSAI WY, DELAPAZ R, MAYEUX R, STERN Y. (2002). Imaging hippocampal function across the human life span: is memory decline normal or not? Ann Neurol 51:290 -295.

STANLEY J. COLCOMBE, KIRK I. ERICKSON, NAFTALI RAZ, ANDREW G. WEBB, NEAL J. COHEN, EDWARD MCAULEY AND ARTHUR F. KRAMER(2003). Aerobic Fitness Reduces Brain Tissue Loss in Aging Humans. The Journals of Gerontology Series A: Biological Sciences and Medical Sciences 58:M176M180.

STANLEY, C. E KRAMER AF. (2003). Fitness effects on the cognitive function of older adults: a metaanalytic study. Psychol Sci. 14, 125-130.

VAN BOXTEL MP, PAAS FG, HOUX PJ, ADAM JJ, TEEKEN JC, JOLLES J. (1997). Aerobic capacity and cognitive performance in a cross-sectional aging study. Med Sci Sports Exerc 29:1357-65.

VAN PRAAG, H.; SHUBERT, T.; ZHAO, C. AND GAGE, F.H. (2005). Exercise Enhances Learning and Hippocampal Neurogenesis in Aged Mice. The Journal of Neuroscience, September 21, 25(38):8680-8685.

YAFFE, KRISTINE; BARNES, DEBORAH, NEVITT, MICHAEL; LUI, LI-YUNG E COVINSKY, KENNETH. (2001). A Prospective Study of Physical Activity and Cognitive Decline in Elderly Women Women Who Walk- Arch Intern Med., 161:1703-1708.

WOOD R, REYES-ALVAREZ R, MARAJ B, METOYER K, WELSCH M.(1999). Physical fitness, cognitive function, and health related style of life in older adults. J Aging Phys Act; 7:217-30.

Persp. online: biol. \& saúde, Campos dos Goytacazes, 6 (2), 35-43, 2012 